\title{
The Influence of Glycosidic Linkage Neighbors on Disaccharide Conformation in Vacuum
}

\author{
${ }^{1,4}$ R. Kramer Campen, ${ }^{2,3}$ Ana Vila Verde, ${ }^{1}$ James D. Kubicki \\ ${ }^{1}$ Department of Geosciences and \\ ${ }^{2}$ Department of Chemical Engineering \\ Pennsylvania State University, \\ University Park, PA 16802 \\ ${ }^{3}$ Centro de Física \\ Universidade do Minho \\ Campus de Gualtar \\ 4710-057 Braga, Portugal \\ ${ }^{4}$ now at \\ FOM Institute for Atomic and Molecular Physics \\ Kruislaan 407 \\ 1098 SJ, Amsterdam, The Netherlands \\ campen@amolf.nl
}

January 3, 2008

\begin{abstract}
Correct description of the free energy of conformation change of disaccharides is important in understanding a variety of biochemical processes and, ultimately, in the manufacture of better food and paper products. In this study we determine the relative free energy of a series of twelve disaccharides in vacuum using replica exchange
\end{abstract}


molecular dynamics (repMD) simulation. The chosen sugars and the novel application of this method allow the exploration of the role of glycosidic linkage neighbors in conformer stabilization. In line with expectations we find that hydrogen bonding (and therefore energetically preferred conformations) are determined both by the nature of the glycosidic linkage (i.e. $1 \rightarrow 2,1 \rightarrow 3$ or $1 \rightarrow 4$ ), the $\mathrm{C} 1$ epimer of the of the nonreducing monosaccharide and the configuration of carbon atoms once removed from the glycosidic linkage. Contrary to suggestions by prior authors for repMD more generally, we also demonstrate that repMD provides enhanced sampling, relative to conventional MD simulations of equivalent length, for disaccharides in vacuum at $300 \mathrm{~K} .^{1}$

Keywords: disaccharide, free energy, replica exchange MD

\section{Introduction}

Homopolysaccharides are ubiquitous in nature functioning both as energy storage molecules and as reinforcements of cellular structures. ${ }^{2}$ Understanding the chemistry of these and other biological functions requires understanding both atomic and molecular level properties of the polysaccharide of interest. On the atomic level the strengths of individual bonds and of the non-bonded interactions of particular functional groups are important in understanding the energy available in these compounds and their solubility; while on the molecular level the flexibility and secondary structure of these molecules determine their macroscopic mechanical properties.

Heteropolysaccharides (often with a repeat unit of four to five) are also used in a variety of organisms for a number of physiological functions. In bacteria, these molecules can be both tethered to the cell surface, as is the case for Gram negative cells (through a lipid anchor inserted in the outer membrane ${ }^{3}$ ), or extruded from cells 
into solution. ${ }^{4}$ Among their functions are cellular recognition, the control of cellular adhesion, the storage of trace nutrients and service as structural elements (i.e. the building blocks) of biofilms. ${ }^{5-8}$ Bacterially produced heteropolysaccharides can also have significant effects on food products (principally dairy) that have live microbial communities. ${ }^{9}$ The detailed description of these systems requires, as is the case for the homopolysaccharides, understanding both atomic and molecular scale properties of the relevant molecules.

There is extensive evidence, both from simulation and experiment, that the homo and heteropolysaccharides of interest (generally constructed of pyranose repeat units) are stiff relative to simpler homo- and heteropolymers. ${ }^{9-13}$ Because individual monosaccharides have limited flexibility (although ring 'flips' are important in some biological contexts $^{14}$ ) the structure of polysaccharides can often be predicted through an understanding of the glycosidic linkage between monosaccharides. These linkages can be fully described by two $(\phi / \psi)$, or in the case of $1 \rightarrow 6$ connections, three $(\phi / \psi / \omega)$, torsion angles (hereafter we consider only $1 \rightarrow 2,1 \rightarrow 3$ and $1 \rightarrow 4$ connections and so address only $\phi$ and $\psi$ ). Analogously to the standard practice in constructing a Ramachandran plot for proteins, it is straightforward to generate a Ramachandran plot for disaccharides, quantifying potential energy in vacuum as a function of $\phi$ and $\psi$. Similar to the protein case, such plots, while directly lending insight into the linkage between monosaccharides, also have broader implication for polysaccharide conformation (e.g. particular ranges of $\phi / \psi$ angles imply particular secondary structural motifs). It is generally implicit in plotting energy as a function of $\phi$ and $\psi$ that these are the most important degrees of freedom in determining relative conformer energies. However, many computational studies have demonstrated, particularly for sugars in vacuum, 
that the conformation of exocyclic groups is energetically important. ${ }^{15,16}$ As a result, in generating a $\phi / \psi$ map much effort is typically expended to achieve the lowest energy conformation of these exocyclic groups for each $\phi / \psi$ point. For maps formed from energy minimized structures this problem is surmounted by minimizing a variety of initial structures. For maps constructed from finite temperature dynamics simulations an enhanced sampling approach is generally necessary. Both these approaches are discussed further below.

Most of the physiological and materials applications alluded to above require the consideration of sugar structure in water at temperatures $\approx 300 \mathrm{~K}$. However, several decades of work has demonstrated the utility of the construction of Ramachandran plots of minimum potential energy as a function of disaccharide glycosidic torsions in vacuum (an approach generally termed the vacuum adiabatic map). ${ }^{17-21}$ Despite their distance from most applications of interest, much effort has been devoted to the construction of adiabatic maps for three reasons. Firstly, from a computational point of view, the vacuum energy minimizations necessary to construct adiabatic maps have the advantage of consuming far fewer resources than more complicated simulations. This lower expense alone clarifies the historical importance of this approach and justifies its continued utility as a means of testing the application of new hardware or potential energy expressions to carbohydrate simulation. Secondly, populated energy minima in classical molecular dynamics simulation, both in vacuum and solution, often appear to roughly follow the adiabatic map (e.g. molecular dynamics simulations of carrabiose and explicitly solvated $\beta$ and $\kappa$-carrageenan ${ }^{22-24}$ ). Finally, adiabatic maps have been shown to be predictive of disaccharide conformation in crystals ${ }^{25}$ and are also clearly important in interpreting results from an increasing number of analytical techniques 
that directly quantify the conformation and relative number of various disaccharide conformers in vacuum. ${ }^{26-29}$

Initial efforts at generating vacuum adiabatic maps employed a rigid body approximation for each monosaccharide: calculating the potential energy using an empirical force field as a function of incremental, independent, rotations of $\phi$ and $\psi \cdot{ }^{17}$ Subsequent methodological improvement led to the creation of 'relaxed' $\phi / \psi$ maps in which all degrees of freedom except the glycosidic torsions are minimized. ${ }^{18-20,25,26}$ Simple conformational searches revealed, early in this work, the existence of 'multiple minima' at each point in $\phi / \psi$ space. ${ }^{30}$ The usual approach to surmounting this problem has been starting minimizations at each $\phi / \psi$ point from a variety of initial structures. ${ }^{26,31}$ The number and nature of the starting structures minimized has generally been informed by available computational resources and chemical intuition, with modern efforts employing $>10$ per $\phi / \psi$ point.

While useful, adiabatic maps suffer from shortcomings in the description of both vacuum and solvated disaccharide dynamics simulation. In the former case, the adiabatic map (assuming a global minimum at each $\phi / \psi$ point has been identified) neglects any influence of conformational entropy on the frequency with which a particular $\phi / \psi$ value will appear in a molecular dynamics trajectory. In the latter case the adiabatic map clearly ignores any enthalpy or entropy of solvation. Consideration of these problems should make clear that what is really of interest is not a potential energy surface (as is described in the adiabatic map) but rather a free energy surface. In this study we calculate such surfaces for several disaccharides in vacuum using the replica exchange molecular dynamics method (repMD). In what follows other ways of creating such surfaces, and the characteristics of repMD that make it a useful alternative, are discussed 
in greater detail.

If all conformational states could be well sampled, a conventional molecular dynamics simulation could, in principle, be used to construct a free energy surface. In practice, however, the disaccharide $\phi / \psi$ potential energy surface has too much variation in energy (with or without the water) to allow adequate sampling using conventional MD in computationally accessible time scales. ${ }^{32}$

This time scale problem is well known in computational chemistry and physics and is typically surmounted with one of two basic strategies: reducing the systems' degrees of freedom (e.g. coarse graining ${ }^{33}$ ), or retaining molecular detail and adapting an enhanced sampling algorithm. ${ }^{34,35}$ Several recent studies have addressed the coarse grained simulation of polysaccharides in concentrated solutions or condensed phases. ${ }^{36-38}$ This approach, however, requires parameterizing the details of the flexibility of the glycosidic linkage and, in the case of the solvated system, the disaccharide interaction with $\mathrm{H}_{2} \mathrm{O}$. Since it is the proper sampling of the glycosidic flexibility and disaccharide interaction with solvent that we are ultimately after, an accelerated sampling strategy that retains atomic detail seems preferable.

In the last ten years Brady, Naidoo and coworkers have addressed this problem by application of adaptive umbrella sampling molecular dynamics to various disaccharides both in solution and in vacuum. ${ }^{32,39-42}$ This approach differs from conventional molecular dynamics in that an extra "umbrella potential" is added to the potential energy expression. This umbrella potential is taken to be a function of $\phi$ and $\psi$ and is chosen so as to render the system diffusive in $\phi / \psi$ space. While rigorous, this approach is computationally demanding to implement because the form of the umbrella potential is not known a priori and as a result must be iteratively refined. 
Adaptive umbrella sampling can be understood as part of a larger class of simulation algorithms that allow enhanced sampling of conformational states by searching phase space with non-Boltzmann probabilities, termed generalized ensemble algorithms. ${ }^{34}$ These methods are attractive because in principle they allow the molecule of interest to sample conformations of widely different energy at a rate limited only by diffusion. Problematically, as prior studies have observed, ${ }^{32,41}$ the determination of the weights necessary to search such phase space diffusively is challenging.

Replica exchange molecular dynamics (repMD) avoids this challenge. In repMD molecular systems diffuse through temperature space and thus (provided the temperature range is sufficiently large) also diffuse through the relevant portion of energy space. $^{35}$ In its molecular dynamics incarnation, this idea is implemented by simultaneously running a series of conventional molecular dynamics simulations, over a range of target temperatures, with the replicas swapping configurations after some elapsed time. This approach gives thermodynamically exact results because detailed balance is maintained during the swapping, typically through use of the Metropolis criterion.

Variously termed replica exchange molecular dynamics and parallel tempering molecular dynamics (hereafter repMD), this algorithm has in the last 10 years been employed in numerous studies of protein folding ${ }^{35,43-50}$ but only once, to our knowledge, in the simulation of sugars. ${ }^{51}$ That study used the enhanced sampling provided by replica exchange MD (there termed "parallel tempering Monte Carlo") to study the water structure in concentrated solutions of sucrose and trehalose near their glass transition temperature.

Decades of experiment and simulation confirm the chemical intuition that disaccharide flexibility is a function both of the anomer of the nonreducing sugar (i.e. $\alpha$ or 
$\beta)$ and the type of connection $(1 \rightarrow 2,1 \rightarrow 3,1 \rightarrow 4$ or $1 \rightarrow 6)$. Less clear is the degree to which epimerization at other positions on either pyranose ring helps determine energetically preferred conformations. Here we address this problem in a systematic way by constructing relative free energy (or potential of mean force: PMF) $\phi / \psi$ surfaces for a series of 12 disaccharides in vacuum (see Table 1 for details). This approach allows us to describe energetically favored conformations and to quantify their relative free energy. We also demonstrate the enhanced sampling that repMD provides for disaccharides in vacuum by comparing the repMD results at $300 \mathrm{~K}$ to results from conventional MD simulations of equivalent length (i.e. the $300 \mathrm{~K}$ results of a repMD simulation containing 6 replicas, each run for $10 \mathrm{~ns}$, are compared to a conventional MD simulation at $300 \mathrm{~K}$ run for $60 \mathrm{~ns})$.

In broad agreement with prior work we find that preferred conformations are stabilized by intermonosaccharide hydrogen bonding. Geometric constraints suggest that such intermonosaccharide hydrogen bonding should proceed largely through neighbors of the glycosidic linkage: e.g. a conformation of $\alpha$-D-Glucose $(1 \rightarrow 2) \alpha$-D-Glucose might be stabilized by some combination of interactions of $\mathrm{O}^{\prime}, \mathrm{O} 22^{\prime}, \mathrm{OH} 2 \prime, \mathrm{O} 1, \mathrm{OH} 1, \mathrm{O} 3$ and OH3 (see Figure 1 for a description of labeling scheme). Our simulations suggest that the degree and type of neighbor involvement is a function of both the epimerization of those carbon atoms involved in the glycosidic linkage and of their neighbors.

Prior studies that have discussed the relationship of hydrogen bonding and disaccharide type in vacuum often approach the problem in one of two ways: by comparing adiabatic maps generated using a disaccharide analog (without the exocyclic groups) to adiabatic maps of the full disaccharide (or experimental $\phi / \psi$ values from crystal structures $)^{52}$ or by examining the adiabatic map of full disaccharides over a range of, 
increasing dielectric constants (see, for example, French et al. ${ }^{21}$ ). Our approach differs from these prior studies in two ways. Firstly, it describes the relative free, rather than the relative potential, energy. Secondly, these prior studies minimize the influence of all hydrogen bonds simultaneously while our approach describes the influence of single small changes in structure on hydrogen bonding.

PMF surfaces of the sort we have described are of use in quantifying the flexibility of di- and polysaccharides in vacuum. This information is important both for its own sake, since increasingly conformer selective analytical techniques can be used to investigate di- and oligosaccharides in vacuum, and also as an intermediate between the description of solvated and condensed phase di- and polysaccharides. This kind of understanding of the free energy of conformation change is critical both in the description of a wide variety of biochemical processes (ranging from cell surface recognition to the mechanical properties of vascular plants) and designing better paper materials. Furthermore the demonstration of the application of repMD for this purpose provides a potentially useful alternative to the computationally intensive adaptive umbrella sampling.

\section{Methods}

The 12 disaccharides employed in this study (see Table 1) were constructed using a web-based carbohydrate builder. ${ }^{53}$ The structures chosen and the model \# by which they are referred to hereafter are shown in Table 1. The atom labeling scheme used in the study is illustrated in Figure 1. We defined the glycosidic torsion angles in this work by reference to non-hydrogen atoms: $\phi=\mathrm{O} 5^{\prime}-\mathrm{C}^{\prime}-\mathrm{On}-\mathrm{Cn}$ and $\psi=\mathrm{C} 1^{\prime}-\mathrm{On}-\mathrm{Cn}-\mathrm{C}(\mathrm{n}+1)$. All simulations were performed using the NAMD program. ${ }^{54}$ All conventional molecular dynamics simulations were run in the canonical ensemble (NVT), with a timestep 
of $1 \mathrm{fs}$ and all $\mathrm{O}-\mathrm{H}$ bonds constrained using the SHAKE algorithm. Nonbonded interactions were calculated using a cutoff of $12 \AA$ with interactions tapered to zero starting at $10 \AA$. Temperature was controlled at $300 \mathrm{~K}$ using a Langevin thermostat with $\gamma=5$ $\mathrm{ps}^{-1}$. The GLYCAM04 parameters were employed and, following the recommendation of their authors, all nonbonded interactions between 1,4 atoms were scaled by $1 .{ }^{55-58}$ Equilibration simulations of 200 ps were run before all production runs. All simulations were sampled every 4 ps. Visualization of all structures was performed using $\mathrm{VMD}^{59}$ and analysis using scripting extensions to VMD and IGOR Pro (Wavemetrics).

RepMD was run in NAMD following a previously described algorithm. ${ }^{35}$ Summarized, our approach was to generate a series of six identical replicas whose target temperatures were 300, 355, 421, 499, 591 and 700 K. Each replica was equilibrated for 200 ps and simulated for a total of 10 ns. All parameters describing each replica simulation - time step, method of non-bonded interaction calculation, thermostat, sampling of trajectory - were the same as for the MD simulations. Swaps between replicas of neighboring target temperature were attempted every 1 ps. Swaps were accepted with a Metropolis criterion: the probability of accepting a swap between replicas running at temperatures i and $\mathrm{i}+1$ was (where $\beta_{i}=\frac{1}{k T_{i}}$ and $E_{i}=$ potential energy of replica at temperature i),

$$
P_{a c c}=\exp \left[\left(\beta_{i}-\beta_{i+1}\right)\left(E_{i}-E_{i+1}\right)\right]
$$

If a swap is accepted the target temperatures of each replica are exchanged, velocities rescaled to the target temperature, and the simulation allowed to continue for another ps. Recent work suggests our choice of 1 ps between attempted replica exchanges is sufficiently long to avoid excessive correlation between acceptances (i.e. exchange two replicas in one attempt and reverse the exchange at the next attempt) but sufficiently 
short to be relatively efficient in searching phase space. ${ }^{50}$

RepMD simulations were run for each disaccharide from multiple starting structures (see Table 2 for a list of initial $\phi / \psi$ values). After each simulation a normalized two dimensional histogram was created from all sampled $\phi / \psi$ values using a $5^{\circ}$ grid. The results of $\phi / \psi$ frequency were normalized and then averaged for all simulations for a particular model. The relative free energy (W) in any grid square can be related to the probability of the system sampling that location, ${ }^{46}$

$$
P\left(\phi_{i}, \psi_{i}\right)=\frac{1}{Z} \exp [-\beta W(\phi, \psi)]
$$

where $P\left(\phi_{i}, \psi_{i}\right)$ is the probability of the system sampling $\phi_{i} / \psi_{i}$ and $\mathrm{Z}$ is the partition function. The PMF (or relative free energy) is then,

$$
W\left(\phi_{i}, \psi_{i}\right)-W\left(\phi_{g m}, \psi_{g m}\right)=-R T \ln \left[\frac{P\left(\phi_{i}, \psi_{i}\right)}{P\left(\phi_{g m}, \psi_{g m}\right)}\right]
$$

in which the subscript $g m$ indicates the global minimum. The PMF plots shown in Figure 2 and 3 are based on the averaged normalized probability histograms from each of the initial structures (see Figure 4 for an illustration of the averaging).

After construction and analysis of the PMF surfaces, hydrogen bond frequency and type was calculated using one repMD simulation for each disaccharide. In calculating these quantities we used two different groups of geometric parameters: a looser definition, after prior authors, ${ }^{60,61}$ in which a hydrogen bond has an $\mathrm{O} \cdots \mathrm{H}-\mathrm{O}$ angle $>140^{\circ}$ and an $\mathrm{O} \cdots \mathrm{O}$ distance $<3.5 \AA$ as well as a more restrictive definition in which a hydrogen bond has an $\mathrm{O} \cdots \mathrm{H}-\mathrm{O}$ angle $>160^{\circ}$ and an $\mathrm{O} \cdots \mathrm{O}$ distance $<3 \AA$. In general, while frequencies of hydrogen bonding are greater for the looser criteria, no significant 
differences were observed for any disaccharide in the atoms involved in hydrogen bonding nor in the relative importance of a given hydrogen bond. It is expected that the criteria we tested may exclude some stabilizing interactions. However, in accord with chemical intuition, much prior work argues that any such interactions not described are likely substantially weaker than those falling within our criteria. Because the number of possible weak interactions is strongly limited by our relatively small system, it seems unlikely that our criteria miss any significant stabilizing effects.

\section{Results and Discussion}

\subsection{Diagnostics for $\operatorname{repMD}$}

RepMD works by having all replicas diffuse through the specified temperature range. If the target temperatures of each replica are too far apart swaps will be infrequent and phase space will be searched poorly. Conversely, if replica target temperatures are too close together, swaps will occur at most attempts and the search of conformational phase space will be inefficient. Using our parameters the acceptance rate of replica swap was $\approx 35 \%$ for all disaccharides studied (see Table 3 for acceptance ratios of a representative simulation) well within the range employed in previous studies.

Similarly, if sampling at a given temperature is sufficient, potential energy histograms as a function of target temperature will be gaussian. Figure 5 shows the potential energy distribution as a function of temperature for one representative simulation. The histograms clearly follow the anticipated trend.

Taken together these metrics suggest that the system is well sampled at particular temperatures and that the replicas readily exchange between temperatures. However, 
this gives no indication as to whether $\phi / \psi$ space is being adequately sampled. Hypothetically, if $\phi / \psi$ values from the high temperature replica in a repMD simulation were random, one could be assured that all energy barriers could be overcome. However, much prior work has demonstrated that steric clashes in $\phi / \psi$ space lead to energies (relative to the global minimum) well in excess of $20 \mathrm{kcal} / \mathrm{mol}$. Roughly doubling temperature does not provide sufficient energy to overcome such barriers. Barrier hopping through higher temperature replicas (in excess of $1000 \mathrm{~K}$ ) with our relatively small systems is algorithmically challenging (integration schemes of the equations of motion become unstable). Fortunately it is also unnecessary as the probability of disaccharides in vacuum or in solution existing in such sterically prohibited conformations is low. In this study we insure that we have reliably sampled all $\phi / \psi$ states accessible at $300 \mathrm{~K}$ by running repMD from three or four widely spaced (in $\phi / \psi$ space) starting structures (see Table 2 for a list of the initial values of $\phi$ and $\psi$ for all repMD simulations of all models and Figure 4 for an illustration of the results as a function of starting structure for one model). For all the disaccharides tested each starting structure resulted in a quantitatively equivalent $\phi / \psi$ distribution. The resulting free energy surfaces (shown in Figures 2 and 3) are averages over the result from all three to four starting structure.

PMF surfaces shown in Figures 2 and 3 each contain substantial amounts of "white space': $\phi / \psi$ values that, within the length of our simulation, each disaccharide has with low probability. Provided that the temperature range of the replicas allows sampling of the entire PES, as is argued by the identical PMF surfaces produced from simulations initiated with different initial values of $\phi$ and $\psi$, this simply implies that at $300 \mathrm{~K}$ and in vacuum these values of $\phi$ and $\psi$ do not contribute to the observed probability density. As might be expected (and as illustrated in Figure 6 for one simulation) a higher 
temperature replica will sample a larger range of $\phi / \psi$ with significant probability. PMF surfaces produced using repMD will necessarily look different than those produced by umbrella sampling molecular dynamics, in which generally, an umbrella potential is chosen iteratively so as to drive an MD simulation that samples all $\phi / \psi$ values within a given range with equal probability (and the relative energies of each state are then back calculated from the value of the umbrella potential). ${ }^{32,40-42}$

\subsection{Comparison of the repMD and conventional MD for searching disaccharide phase space}

Zuckerman et al. ${ }^{1}$ have recently suggested that the repMD algorithm is unlikely to give appreciable increases in computational efficiency vs. conventional molecular dynamics for systems in which barrier heights are relatively small - as may be typical for biopolymers in solution. They arrive at this conclusion by assuming that the rate-limiting step in searching phase space in biomolecular simulation can be represented as an Arrhenius type energy barrier. Given this assumption it is straightforward to show that a repMD scheme does not change the prefactor (the entropic part) and the influence on the energy portion of the equation for small barriers is minimal. The consequence of this argument is that the algorithm of repMD would only lead to enhanced sampling in cases in which barriers are suitably large.

It seems reasonable to believe that the energy barriers to conformational change in vacuum would be larger than those in solution and thus, perhaps, sufficiently large to lead to enhanced searching of phase space in repMD vs conventional MD. In any case, more recently Periole et al. have argued that the rate of phase space searching for a large peptide in water is not a function of barrier crossing, but rather the rate 
at which the peptide experiences small, lower energy, phase changes, suggesting that repMD may lead to enhanced sampling even in the absence of particularly high energy barriers. $^{50}$

In this study we address this issue empirically, by comparing the sampling that repMD simulations offer of various degrees of freedom with the sampling of equivalent length conventional MD simulations. Before discussing these results, it is worth noting that the speed up (or lack thereof) referenced by Zuckerman et al. in searching phase space using repMD is only algorithmic. Because only temperatures are passed between replicas, shorter repMD simulations will, on virtually all modern clusters, have the equivalent hardware cost of a single longer MD simulation (whose length is the sum of the lengths of each replica simulation).

In terms of searching $\phi / \psi$ space we find generally that, for disaccharides with two separated minima, sampling of a $10 \mathrm{~ns}$ repMD simulation is superior to a $60 \mathrm{~ns}$ conventional MD simulation (see Figure 7). Similarly comparison of the sampling of dihedrals describing the orientation of exocyclic hydroxyl groups suggests that for this degree of freedom sampling is enhanced in the repMD approach as well (a representative example is shown in Figure 8). In contrast, the sampling of hydroxymethyl conformations, as shown in Figure 9, appears to be roughly equivalent in the two approaches, arguing that for this degree of freedom repMD gives little advantage.

\subsection{Relative Free Energy}

Relative free energy as a function of $\phi / \psi$ is shown for the $\alpha(1 \rightarrow 2), \alpha(1 \rightarrow 3)$ and $\alpha(1 \rightarrow 4)$ connected disaccharides in Figure 2 and for the $\beta(1 \rightarrow 2), \beta(1 \rightarrow 3)$ and $\beta(1 \rightarrow 4)$ in Figure 3. In addition, the location and relative magnitude of the vacuum adiabatic minima 
for the six glucose dimers from Mendonca et al. are also shown (in that work they use different anomers of the reducing monosaccharide for models 4, 5 and 6). ${ }^{26}$ In general, we have included minima identified by Mendonca et al. that are up to $3.5 \mathrm{kcal} / \mathrm{mol}$ above their global minima. Minima of higher relative energy would be expected to be relatively unpopulated at $300 \mathrm{~K}$ (at which $\mathrm{kT} \approx 0.6 \mathrm{kcal} / \mathrm{mol}$ ). In addition to the PMF plots, hydrogen bonds observed (in one repMD run) for each model and their frequency are indicated in Table 4. It is worth noting that the adiabatic maps of Mendonca et al. were constructed using the MM3 force field (as opposed to the GLYCAM in our study). Differences in potential functions are known to cause differences both in adiabatic maps and other computed sugar properties. ${ }^{62}$ As is discussed below, in general we find our results in good agreement with those of Mendonca et al. In those cases where the two studies disagree differences are generally rationalizable by noting the different characteristics of a vacuum adiabatic map and a PMF surface. For this reason we believe that differences between the studies reflect differences in the physical basis of a PMF surface as compared to that of the adiabatic map and do not discuss the consequence of the different potential functions employed further.

Because the glycosidic linkage in models 1 and 7 is $\alpha(1 \rightarrow 2)$, and because the models differ only in configuration at $\mathrm{O}^{\prime}$ and $\mathrm{O} 4$ we might expect the PMFs, as a function of $\psi$ and $\phi$, to be equivalent. As Figure 2 makes clear, this is indeed the case. Further, we also find the PMF surface of both models is in reasonable agreement with minima derived from the adiabatic vacuum map (dielectric constant $=1.5$ ) of Mendonca et al. ${ }^{26}$ Such agreement implies that the role of conformational entropy is relatively minimal for these models. The general pattern of the PMF, namely $\phi$ values of $\approx$ $100^{\circ}$, is consistent with a role of the exo-anomeric effect in stabilizing each of these 
conformers. Analysis of the inter-saccharide hydrogen bonding suggests that both models also experience similar hydrogen bonds with similar frequency (see Table 4). Correlation of the hydrogen bonds' geometric parameters with $\psi$ suggests that each minimum, for each model, is stabilized by different $\mathrm{H}$ bonds (see Figure 10).

The glycosidic bond of models 2 and 8 is $\alpha(1 \rightarrow 3)$. As for models 1 and 7,2 and 8 differ only in the configuration of $\mathrm{O}^{\prime}-\mathrm{OH} 4^{\prime}$ and $\mathrm{O} 4-\mathrm{OH} 4$ relative to the ring to which they are attached. Inspection of the PMF surfaces suggests that while both have one minimum located in the same general area in $\phi / \psi$ space, the details surrounding this minimum differ. As for models 1 and 7 the location of this populated minimum is near, but not equivalent to, the two lowest energy minima identified in the vacuum adiabatic map by Mendonca et al. Inspection of hydrogen bonding suggests that, while both models are stabilized by the same interactions, the role of the $\mathrm{O} 2^{\prime} \cdots \mathrm{OH} 4-\mathrm{O} 4$ interaction is substantially more important for the galactose dimer (model 8) than the glucose (model 2), while the $\mathrm{O}^{\prime} \cdots \mathrm{OH} 6-\mathrm{O} 6$ behaves oppositely. Differences in the intensity of the former interaction between models 2 and 8 are in line with expectations - in model 2 the $\mathrm{OH} 4-\mathrm{O} 4$ and $\mathrm{O} 3-\mathrm{C} 3$ groups are oriented similarly with respect to the reducing monosaccharide ring, while in model 8 they are oriented differently. Additionally, it is worth pointing out that the disagreement between the Mendonca et al. results and those for model 2 likely springs, at least in part, from conformational entropy. Inspection of Figure 11 shows that in model $2 \psi$ values between $75^{\circ}$ and $125^{\circ}$ are predominantly stabilized both by a $2^{\prime} \ldots \mathrm{OH} 6-\mathrm{O} 6$ interaction and a $\mathrm{O}^{\prime} \cdots \mathrm{OH} 4-$ O4 interaction. The overlap of two distinct stabilizing interactions in $\psi$ space, both involving the same H-bond acceptor, is missed in the vacuum adiabatic approach.

Models 3 and 9 both have a glycosidic bond that is $\alpha(1 \rightarrow 4)$. However, because the 
two models are $\mathrm{C} 4$ epimers it seems reasonable to expect that they should generate markedly different PMF surfaces. While both surfaces have a $\phi$ value centered around $+100^{\circ}$, a value consistent with the exo-anomeric effect, the values of $\psi$ differ by $\approx$ $60^{\circ}$. The different value of $\psi$ can be rationalized by noting that, given the difference in configuration of the $\mathrm{O} 4-\mathrm{C} 4$ group between the two monosaccharides, this change in $\psi$ serves to minimize steric conflicts between the two monomers. Given the change in configuration of the linkage between the two monosaccharides, differences in hydrogen bonding are expected. As shown in Table 4, the two models do not share any hydrogen bonds. It is also apparent in the hydrogen bonding pattern that model 3 has only one significant interaction. The existence of a single significant stabilizing interaction might be thought to minimize the influence of conformational entropy on this structure, and indeed the PMF global minimum nearly perfectly reproduces the location of the vacuum adiabatic global minimum. It should also be noted that prior analyses of PMF surfaces of model 3 (i.e. maltose) constructed using umbrella sampling MD with a different forcefield from that employed either by us or Mendonca et al. give a global minima in essential agreement (within the $0.50 \mathrm{kcal} / \mathrm{mol}$ contour on our surface). ${ }^{42}$

Models 4 and 10 both have a $\beta(1 \rightarrow 2)$ glycosidic bond. As is the case for models 1 and 7 these structures differ only in the configuration of the $\mathrm{O}^{\prime}-\mathrm{OH} 4^{\prime}$ and $\mathrm{O} 4-\mathrm{OH} 4$ groups. As these groups are not close to the glycosidic linkage it might be anticipated that their $\phi / \psi$ surfaces should be largely the same. As reference to Figure 3 makes clear, this is indeed the case. Moreover, for both models the great majority of sampled conformers have a $\phi$ value of $\approx-100^{\circ}$, consistent with the exo-anomeric effect for $\beta$ linked sugars. As might be expected for models with similar PMF surfaces, intermonosaccharide hydrogen bonds stabilizing the minima are the same (model 4 enjoys 
marginally more flexibility and in this case an exchange of the donor and acceptor in one interaction are observed). In the case of model 4, our PMF surfaces reproduce the global minimum of Mendonca et al. ${ }^{26}$ but do not noticeably capture any of the other low energy conformers. One possible explanation for this discrepancy may lie in the slight structural difference (the anomeric state of the reducing monosaccharide) between our model ( $\alpha$-sophorose) and the sophorose studied by Mendonca et al. However, an alternative explanation is that the secondary, tertiary and quaternary minima they observe in this molecule are either un- or only weakly stabilized by hydrogen bonding. It seems possible, therefore, that these conformers may simply be unstable at finite temperatures. It should be emphasized in this case that our inability to sample the sophorose secondary minima of Mendonca et al. in particular is almost certainly not an artifact of searching phase space poorly as two initial structures for repMD analyses were substantially closer to this point in $\phi / \psi$ space than to the global minimum.

Models 5 and 11 both have a $\beta(1 \rightarrow 3)$ glycosidic linkage. As is the case for all other pairs of models described, these models differ only in the configuration of the $\mathrm{OH} 4^{\prime}-\mathrm{O} 4^{\prime}$ and $\mathrm{OH} 4-\mathrm{O} 4$ groups. Because this difference adjoins the glycosidic linkage it seems reasonable to anticipate that it may play a significant role in determining which regions of the PMF surfaces are populated. In general, the populated regions of the PMF surfaces are similar to those previously noted in the comparison of models 2 and 8: $\phi$ angles are $\approx-100^{\circ}$, consistent with the exoanomeric effect. The notable exception lies in the global minimum in model 5 (near $\phi=50^{\circ}$ ) which is the secondary minimum in the adiabatic map. As is clear from Figure 12 and Table 4 this minimum

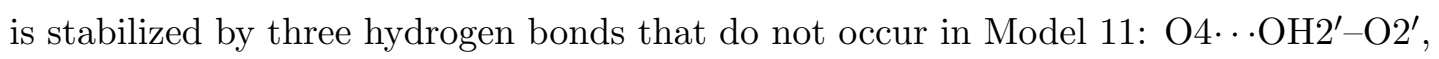
$\mathrm{O}^{\prime} \cdots \mathrm{OH} 4-\mathrm{O} 4$ and $\mathrm{O} 5^{\prime} \cdots \mathrm{OH} 2-\mathrm{O} 2$. Models 5 and 11 differ in the configuration of the 
O4-C4 group. In model 5 this group has a similar configuration to the O3-C3 while in model 11 it does not. These results suggest that orienting the O4-C4 group similarly to the O3-C3 allows several inter-monosaccharide hydrogen bonds not otherwise possible.

Models 6 and 12, both $\beta(1 \rightarrow 4)$ linked, have PMF surfaces similar to the $\alpha(1 \rightarrow 4)$ linked pair, models 3 and 9. For both models 6 and 12 the principal minimum has a $\phi$ value of $\approx-100^{\circ}$ (see Figure 3 ) a result consistent with the exo-anomeric effect. In the case of model 6 the $\psi$ value is $\approx-150^{\circ}$ while for model 12 it is $\approx 160^{\circ}$ both results of which are rationalizable by an attempt to minimize steric clashes between the two rings. The configuration of the glycosidic linkage differs between the two models so, as expected, the intermonosaccharide hydrogen bonding also differs (see Table 4).

\section{Conclusions}

In this work we describe the application of repMD to the construction of maps of PMF as a function of glycosidic torsion angle for a series of twelve disaccharides. We empirically demonstrate that the technique is algorithmically superior to long conventional MD calculations (relative rate of sampling of relevant states as a function of total time simulated) in addition to its usual computational superiority.

Comparison of the PMF maps to vacuum adiabatic maps of the same molecules gives reasonable agreement. However, the vacuum adiabatic approach does not capture the effects of finite temperature and of conformational entropy on the relative energies of different conformers, both effects of which are likely to be important in the description of PMF surfaces for some disaccharides in vacuum.

Finally, we apply these repMD generated surfaces to study the role of the epimerization of carbon atoms adjacent to the glycosidic linkage in the existence of stable 
intermonosaccharide hydrogen bonds. Comparison of $\alpha(1 \rightarrow 2)$ and $\beta(1 \rightarrow 2)$ linked glucose and galactose dimers (models $1 / 7$ and 4/9 respectively) suggests that for each pair, minima in the PMF are stabilized by the same hydrogen bonds: changing the configuration of $\mathrm{OH} 4^{\prime}-\mathrm{O} 4^{\prime}$ and $\mathrm{OH} 4-\mathrm{O} 4$ groups has little influence on hydrogen bonding in $(1 \rightarrow 2)$ linked disaccharides. In contrast, while $\alpha(1 \rightarrow 3)$ linked glucose and galactose dimers (models 2 and 8 ) show little difference in hydrogen bonding, $\beta(1 \rightarrow 3)$ linked glucose and galactose dimers clearly do (models 5 and 11). This contrast suggests that $\mathrm{C} 4$ epimerization is much more important for hydrogen bonding in $\beta(1 \rightarrow 3)$ linked sugars than for $\alpha(1 \rightarrow 3)$. Finally, comparison of hydrogen bonding patterns in $\alpha(1 \rightarrow 4)$ and $\beta(1 \rightarrow 4$ ) linked glucose and galactose dimers (models $3 / 9$ and $6 / 12$ ) indicates, as expected, very little similarity.

In this work we demonstrate the repMD can be usefully applied to understand the thermodynamics of conformational change of disaccharides in vacuum. The description of PMF surfaces in vacuum is of use both in the understanding of disaccharide features in vacuum-based analytical applications ${ }^{26,29}$ and also as an initial description of the behavior of these molecules in aqueous solutions and condensed phases.

Because of the relative stiffness of the glycosidic linkage, we further expect that these results will be useful in describing polysaccharide properties both in solution and in two- and three-dimensional condensed phases through Monte Carlo growth methods. For calculation of properties of di-, oligo- and polysaccharide systems not computed here, our results suggest the application of repMD may be a promising new alternative. In short the results presented in this study should be useful to investigators interested in such diverse topics as basic physical chemistry of disaccharides, paper products, plant physiology, the chemistry of dairy products and the bacterial cell surface. 


\section{Acknowledgements}

This work was supported by the National Science Foundation under Grant No. CHE0431328. AVV acknowledges the Portuguese National Science Foundation (FCT) for post doctoral fellowship SFRH/BPD/20555/2004/0GVL. The comments of two anonymous reviewers greatly helped in improving the manuscript. Their aid is gratefully acknowledged. 
Table 1: Disaccharides simulated and model numbers by which they are referenced in the text.

\begin{tabular}{|c|c|c|}
\hline Model \# & Common Name & Systematic Notation \\
\hline 1 & kojibiose & $\alpha$-D-Glucose $(1 \rightarrow 2) \alpha$-D-Glucose \\
2 & nigerose & $\alpha$-D-Glucose $(1 \rightarrow 3) \alpha$-D-Glucose \\
3 & maltose & $\alpha$-D-Glucose $(1 \rightarrow 4) \alpha$-D-Glucose \\
4 & $\alpha$-sophorose & $\beta$-D-Glucose $(1 \rightarrow 2) \alpha$-D-Glucose \\
5 & $\alpha$-laminarabiose & $\beta$-D-Glucose $(1 \rightarrow 3) \alpha$-D-Glucose \\
6 & $\alpha$-cellobiose & $\beta$-D-Glucose $(1 \rightarrow 4) \alpha$-D-Glucose \\
7 & - & $\alpha$-D-Galactose $(1 \rightarrow 2) \alpha$-D-Galactose \\
8 & - & $\alpha$-D-Galactose $(1 \rightarrow 3) \alpha$-D-Galactose \\
9 & galabiose & $\alpha$-D-Galactose $(1 \rightarrow 4) \alpha$-D-Galactose \\
10 & - & $\beta$-D-Galactose $(1 \rightarrow 2) \alpha$-D-Galactose \\
11 & - & $\beta$-D-Galactose $(1 \rightarrow 3) \alpha$-D-Galactose \\
12 & - & $\beta$-D-Galactose $(1 \rightarrow 4) \alpha$-D-Galactose \\
\hline
\end{tabular}


Table 2: Initial $\phi / \psi$ values for repMD simulations for each model.

\begin{tabular}{||c||c|c||c||c|c||}
\hline Model \# & $\phi$ & $\psi$ & Model \# & $\phi$ & $\psi$ \\
\hline 1 & 74.28 & -136.09 & 7 & 71.28 & -137.25 \\
& -86.85 & -126.21 & & -100.69 & -125.68 \\
& 73.63 & 129.69 & & 118.8 & 35.21 \\
\hline 2 & 73.74 & 105.18 & 8 & 72.36 & 103.24 \\
& -143.76 & 148.89 & & -84.01 & 121.81 \\
& 58.88 & -119.77 & & 86.71 & -85.71 \\
\hline 3 & 75.08 & -143.01 & 9 & 74.62 & 105.41 \\
& -78.14 & -130.21 & & -116.77 & 124.31 \\
& 68.46 & 28.7 & & 110.23 & -33.76 \\
& 52.4 & 148.8 & & & \\
\hline \multirow{3}{*}{4} & -58.14 & -124.66 & 10 & -59.07 & -125.15 \\
& 117.84 & -134.54 & & 135.85 & -120.26 \\
& -77.99 & 34.03 & & -79.17 & 43.54 \\
& 63.91 & -55.41 & & & \\
\hline 5 & -62.81 & 123.24 & 11 & -61.18 & 117.77 \\
& 144.58 & 89.99 & & 134.24 & 114.08 \\
& -81.23 & -103.49 & & -123.63 & -61.32 \\
& -59.61 & -16.88 & & & \\
\hline 6 & -63.51 & -116.01 & 12 & -62.67 & 121.43 \\
& 150.52 & -149.57 & & 99.08 & 149.03 \\
& -109.55 & 54.53 & & -76.18 & -22 \\
\hline
\end{tabular}


Table 3: Acceptance ratios for a representative repMD simulation (in this case one of model $3)$.

\begin{tabular}{|c|c|}
\hline Target Temperature & Acceptance Ratio \\
\hline \hline $\mathrm{T}_{300}-\mathrm{T}_{355}$ & 0.3522 \\
$\mathrm{~T}_{355}-\mathrm{T}_{421}$ & 0.3436 \\
$\mathrm{~T}_{421}-\mathrm{T}_{499}$ & 0.3246 \\
$\mathrm{~T}_{499}-\mathrm{T}_{591}$ & 0.3366 \\
$\mathrm{~T}_{591}-\mathrm{T}_{700}$ & 0.3296 \\
\hline
\end{tabular}


Table 4: Hydrogen Bond Frequency for each model for one repMD simulation assuming a cutoff of $<3 \AA(<3.5 \AA)$ in $\mathrm{O} \cdots \mathrm{O}$ distance and $>160^{\circ}\left(>140^{\circ}\right)$ in $\mathrm{O} \cdots \mathrm{H}-\mathrm{O}$ angle - looser criteria appear in ().

\begin{tabular}{|c|c|c|c|c|c|}
\hline Model \# & Hydrogen Bond & Frequency & Model \# & Hydrogen Bond & Frequency \\
\hline \multirow[t]{3}{*}{1} & $\mathrm{O}^{\prime} \cdots \mathrm{OH} 3-\mathrm{O} 3$ & $0.21(0.47)$ & \multirow[t]{3}{*}{7} & $\mathrm{O}^{\prime} \cdots \mathrm{OH} 3-\mathrm{O} 3$ & $0.17(0.39)$ \\
\hline & $\mathrm{O} 2^{\prime} \ldots \mathrm{OH} 3-\mathrm{O} 3$ & $0.05(0.10)$ & & $\mathrm{O} 2^{\prime} \cdots \mathrm{OH} 3-\mathrm{O} 3$ & $0.06(0.14)$ \\
\hline & $\mathrm{O} 2^{\prime} \ldots \mathrm{OH} 1-\mathrm{O} 1$ & $0.04(0.14)$ & & $\mathrm{O} 2^{\prime} \cdots \mathrm{OH} 1-\mathrm{O} 1$ & $0.05(0.12)$ \\
\hline \multirow[t]{4}{*}{2} & $\mathrm{O} 2^{\prime} \cdots \mathrm{OH} 6-\mathrm{O} 6$ & $0.19(0.40)$ & \multirow[t]{4}{*}{8} & $\mathrm{O} 2^{\prime} \cdots \mathrm{OH} 4-\mathrm{O} 4$ & $0.21(0.50)$ \\
\hline & $\mathrm{O} 2^{\prime} \cdots \mathrm{OH} 4-\mathrm{O} 4$ & $0.13(0.30)$ & & $\mathrm{O} 2^{\prime} \cdots \mathrm{OH} 6-\mathrm{O} 6$ & $0.04(0.11)$ \\
\hline & $\mathrm{O} 5^{\prime} \cdots \mathrm{OH} 2-\mathrm{O} 2$ & $0.05(0.17)$ & & $\mathrm{O} 5^{\prime} \cdots \mathrm{OH} 2-\mathrm{O} 2$ & $0.04(0.07)$ \\
\hline & $\mathrm{O} 6 \cdots \mathrm{OH} 2^{\prime}-\mathrm{O} 2^{\prime}$ & $0.04(0.09)$ & & & \\
\hline \multirow[t]{3}{*}{3} & $\mathrm{O}^{\prime} \cdots \mathrm{OH} 3-\mathrm{O} 3$ & $0.49(0.89)$ & \multirow[t]{3}{*}{9} & $\mathrm{O} 5^{\prime} \cdots \mathrm{OH} 3-\mathrm{O} 3$ & $0.35(0.60)$ \\
\hline & & & & $\mathrm{O} 6 \cdots \mathrm{OH} 2^{\prime}-\mathrm{O} 2^{\prime}$ & $0.17(0.32)$ \\
\hline & & & & $\mathrm{O} 2^{\prime} \cdots \mathrm{OH} 6-\mathrm{O} 6$ & $0.11(0.27)$ \\
\hline \multirow[t]{2}{*}{4} & $\mathrm{O} 2^{\prime} \cdots \mathrm{OH} 3-\mathrm{O} 3$ & $0.22(0.42)$ & \multirow[t]{2}{*}{10} & \multirow[t]{2}{*}{$\mathrm{O} 2^{\prime} \cdots \mathrm{OH} 3-\mathrm{O} 3$} & \multirow[t]{2}{*}{$0.31(0.63)$} \\
\hline & $\mathrm{O} 3 \cdots \mathrm{OH} 2^{\prime}-\mathrm{O} 2^{\prime}$ & $0.04(0.09)$ & & & \\
\hline \multirow[t]{4}{*}{5} & $\mathrm{O} 2^{\prime} \cdots \mathrm{OH} 2-\mathrm{O} 2$ & $0.13(0.29)$ & \multirow[t]{4}{*}{11} & $\mathrm{O} 2^{\prime} \cdots \mathrm{OH} 2-\mathrm{O} 2$ & $0.21(0.38)$ \\
\hline & $\mathrm{O} 4 \cdots \mathrm{OH} 2^{\prime}-\mathrm{O} 2^{\prime}$ & $0.09(0.23)$ & & $\mathrm{O} 2 \cdots \mathrm{OH} 2^{\prime}-\mathrm{O} 2^{\prime}$ & $0.04(0.09)$ \\
\hline & $\mathrm{O} 5^{\prime} \cdots \mathrm{OH} 4-\mathrm{O} 4$ & $0.06(0.25)$ & & & \\
\hline & $\mathrm{O} 5^{\prime} \cdots \mathrm{OH} 2-\mathrm{O} 2$ & $0.05(0.19)$ & & & \\
\hline \multirow[t]{3}{*}{6} & $\mathrm{O}^{\prime} \cdots \mathrm{OH} 3-\mathrm{O} 3$ & $0.21(0.60)$ & \multirow[t]{3}{*}{12} & $\mathrm{O} 3 \cdots \mathrm{OH} 2^{\prime}-\mathrm{O} 2^{\prime}$ & $0.19(0.33)$ \\
\hline & $\mathrm{O} 6 \cdots \mathrm{OH} 2^{\prime}-\mathrm{O} 2^{\prime}$ & $0.12(0.35)$ & & $\mathrm{O} 2^{\prime} \cdots \mathrm{OH} 3-\mathrm{O} 3$ & $0.14(0.33)$ \\
\hline & $\mathrm{O} 3 \cdots \mathrm{OH} 2^{\prime}-\mathrm{O} 2^{\prime}$ & $0.05(0.12)$ & & & \\
\hline
\end{tabular}




\section{References}

1. Zuckerman, D. M.; Lyman, E. Journal of Chemical Theory and Computation 2006, 2, 1200-1202.

2. Danishefsky, I.; Whistler, R. L.; Bettelheim, F. A. In The Carbohydrates: Chemistry and Biochemistry; Pigman, W.; Horton, D., Eds.; Academic Press: New York, 1970, Vol. IIA, pp 375-412.

3. Beveridge, T. J. Canadian Journal of Microbiology 1988, 34, 363-372.

4. Wingender, J.; Neu, T. R.; Flemming, H.-C. In Microbial Extracellular Polymeric Substances; Wingender, J.; Neu, T. R.; Flemming, H.-., Eds.; Springer-Verlag: Berlin, 1999.

5. Razatos, A.; Ong, Y.-L.; Sharma, M. M.; Giorgiou, G. Proceedings of the National Academy of Sciences of the United States of America 1998, 95, 11059-11064.

6. Wicken, A. J.; Knox, K. W. Biochimica et Biophysica Acta 1980, 604, 1-26.

7. Christensen, B. E.; Characklis, W. G. In Biofilms; Characklis, W. G.; Marshall, K. C., Eds.; John Wiley and Sons: New York, 1990.

8. Danese, P. N.; Pratt, L. A.; Kolter, R. Journal of Bacteriology 2000, 182, 35933596 .

9. van Kuik, J. A.; Vincent, S. J. F.; Leeflang, B. R.; Kroon-Batenburg, L. M. J.; Kamerling, J. P. Canadian Journal of Chemistry-Revue Canadienne De Chimie 2006, 84, 730-742.

10. Matsuo, K. Macromolecules 1984, 17, 449-452.

11. Dais, P.; Marchessault, R. H. Macromolecules 1991, 24, 4611-4614.

12. Kroon-Batenburg, L. M. J.; Kruiskamp, P. H.; Vliegenthart, J. F. G.; Kroon, J. Journal of Physical Chemistry B 1997, 101, 8454-8459.

13. Camesano, T. A.; Wilkinson, K. J. Biomacromolecules 2001, 2, 1184-1191.

14. Lee, G.; Nowak, W.; Jaroniec, J.; Zhang, Q. M.; Marszalek, P. E. Biophysical Journal 2004, 87, 1456-1465.

15. French, A. D.; Johnson, G. P. Canadian Journal of Chemistry-Revue Canadienne De Chimie 2006, 84, 603-612.

16. Schnupf, U.; Willett, J. L.; Bosma, W. B.; Momany, F. A. Carbohydrate Research 2007, 342, 196-216.

17. Brant, D. A. Annual Review of Biophysics and Bioengineering 1972, 1, 369-408.

18. French, A. D. Biopolymers 1988, 27, 1519-1525. 
19. Ha, S. N.; Madsen, L. J.; Brady, J. W. Biopolymers 1988, 27, 1927-1952.

20. Tran, V. H.; Brady, J. W. Biopolymers 1990, 29, 961-976.

21. French, A. D.; Kelterer, A. M.; Johnson, G. P.; Dowd, M. K.; Cramer, C. J. Journal of Molecular Graphics $\&$ Modelling 2000, 18, 95-107.

22. Ueda, K.; Brady, J. W. Biopolymers 1997, 41, 323-330.

23. Ueda, K.; Imamura, A.; Brady, J. W. Journal of Physical Chemistry A 1998, 102, 2749-2758.

24. Ueda, K.; Saiki, M.; Brady, J. W. Journal of Physical Chemistry B 2001, 105, 8629-8638.

25. French, A. D.; Kelterer, A.-M.; Cramer, C. J.; Johnson, G. P.; Dowd, M. K. Carbohydrate Research 2000, 326, 305-322.

26. Mendonca, S.; Johnson, G. P.; French, A. D.; Laine, R. A. Journal of Physical Chemistry A 2002, 106, 4115-4124.

27. Jockusch, R. A.; Kroemer, R. T.; Talbot, F. O.; Snoek, L. C.; Çarçabal, P.; Simons, J. P.; Havenith, M.; Bakker, J. M.; Compagnon, I.; Meijer, G.; von Helden, G. Journal of the American Chemical Society 2004, 126, 5709-5714.

28. Çarçabal, P.; Jockusch, R. A.; Hünig, I.; Snoek, L. C.; Kroemer, R. T.; Davis, B. G.; Gamblin, D. P.; Compagnon, I.; Oomens, J.; Simons, J. P. Journal of the American Chemical Society 2005, 127, 11414-11425.

29. Simons, J. P.; Jockusch, R. A.; Çarçabal, P.; Hung, I.; Kroemer, R. T.; Macleod, N. A.; Snoek, L. C. International Reviews in Physical Chemistry 2005, 24, 489-531.

30. Scheraga, H. A. Biopolymers 1983, 22, 1-14.

31. Stortz, C. A. Carbohydrate Research 1999, 322, 77-86.

32. Naidoo, K. J.; Brady, J. W. Journal of the American Chemical Society 1999, 121, $2244-2252$.

33. Tozzini, V. Current Opinion in Structural Biology 2005, 15, 144-150.

34. Hansmann, U. H. E.; Okamoto, Y. Journal of Computational Chemistry 1997, 18, 920-933.

35. Sugita, Y.; Okamoto, Y. Chemical Physics Letters 1999, 314, 141-151.

36. Molinero, V.; Cagin, T.; Goddard, W. A. Journal of Physical Chemistry A 2004, 108, 3699-3712.

37. Molinero, V.; Goddard, W. A. Journal of Physical Chemistry B 2004, 108, 14141427. 
38. Queyroy, S.; Neyertz, S.; Brown, D.; Muller-Plathe, F. Macromolecules 2004, 37, 7338-7350.

39. Schmidt, R. K.; Teo, B.; Brady, J. W. Journal of Physical Chemistry 1995, 99, 11339-11343.

40. Kuttel, M.; Brady, J. W.; Naidoo, K. J. Journal of Computational Chemistry 2002, 23, 1236-1243.

41. Kuttel, M. M.; Naidoo, K. J. Carbohydrate Research 2005, 340, 875-879.

42. Kuttel, M. M.; Naidoo, K. J. Journal of Physical Chemistry B 2005, 109, 74687474 .

43. Zhou, R. H.; Berne, B. J.; Germain, R. Proceedings of the National Academy of Sciences of the United States of America 2001, 98, 14931-14936.

44. Sanbonmatsu, K. Y.; Garcia, A. E. Proteins-Structure Function and Genetics 2002, 46, 225-234.

45. Zhou, R. H.; Berne, B. J. Proceedings of the National Academy of Sciences of the United States of America 2002, 99, 12777-12782.

46. Zhou, R. Proceedings of the National Academy of Sciences of the United States of America 2003, 100, 13280-13285.

47. García, A. E.; Onuchic, J. N. Proceedings of the National Academy of Sciences of the United States of America 2003, 100, 13898-13903.

48. Rhee, Y. M.; Pande, V. S. Biophysical Journal 2003, 84, 775-786.

49. Liu, P.; Kim, B.; Friesner, R. A.; Berne, B. J. Proceedings of the National Academy of Sciences of the United States of America 2005, 102, 13749-13754.

50. Periole, X.; Mark, A. E. Journal of Chemical Physics 2007, 126, year, article \# 014903.

51. Ekdawi-Sever, N. C.; Conrad, P. B.; de Pablo, J. J. Journal of Physical Chemistry A 2001, 105, 734-742.

52. French, A. D.; Kelterer, A.-M.; Johnson, G. P.; Dowd, M. K.; Cramer, C. J. Journal of Computational Chemistry 2001, 22, 65-78.

53. Woods Group, Biomolecule Builder, GLYCAM Web, Complex Carbohydrate Research Center, The University of Georgia, 12/11/2006.

54. Phillips, J. C.; Braun, R.; Wang, W.; Gumbart, J.; Tajkhorshid, E.; Villa, E.; Chipot, C.; Skeel, R. D.; Kale, L.; Schulten, K. Journal of Computational Chemistry 2005, 26, 1781-1802.

55. Woods, R. J.; Dwek, R. A.; Edge, C. J.; Frasier-Reid, B. Journal of Physical Chemistry 1995, 99, 3832-3846. 
56. Woods, R. J.; Chapelle, R. Journal of Molecular Structure: Theochem 2000, 527, 149-156.

57. Basma, M.; Sundara, S.; Calgan, D.; Vernali, T.; Woods, R. J. Journal of Computational Chemistry 2001, 22, 1125-1137.

58. Kirschner, K. N.; Woods, R. J. Proceedings of the National Academy of Science of the United States of America 2001, 98, 10541-10545.

59. Humphrey, W.; Dalke, A.; Schulten, K. Journal of Molecular Graphics 1996, 14, $33-38$.

60. Luzar, A.; Chandler, D. Nature 1996, 379, 55-57.

61. Chowdhuri, S.; Chandra, A. Physical Review E 2002, 66, article \# 041203.

62. Pérez, S. et al. Carbohydrate Research 1998, 314, 141-155. 


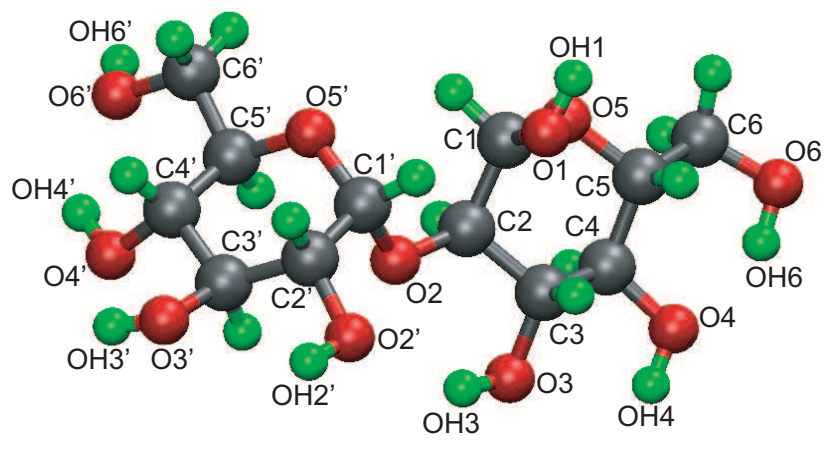

Figure 1: $\alpha$-D-Glucose $(1 \rightarrow 2) \alpha$-D-Glucose, i.e. model 1, indicating numbering scheme. For this model $\phi=\mathrm{O}^{\prime}-\mathrm{C}^{\prime}-\mathrm{O} 2-\mathrm{C} 2$ and $\psi=\mathrm{C} 1^{\prime}-\mathrm{O} 2-\mathrm{C} 2-\mathrm{C} 3$ (in general $\phi=\mathrm{O} 5^{\prime}-\mathrm{C}^{\prime}-\mathrm{O} n-\mathrm{Cn}$ and $\psi=\mathrm{C}^{\prime}-\mathrm{On}-\mathrm{Cn}-\mathrm{C}(\mathrm{n}+1)$ where $\mathrm{n}$ is the number of the Carbon on the reducing ring involved in the glycosidic linkage.). 


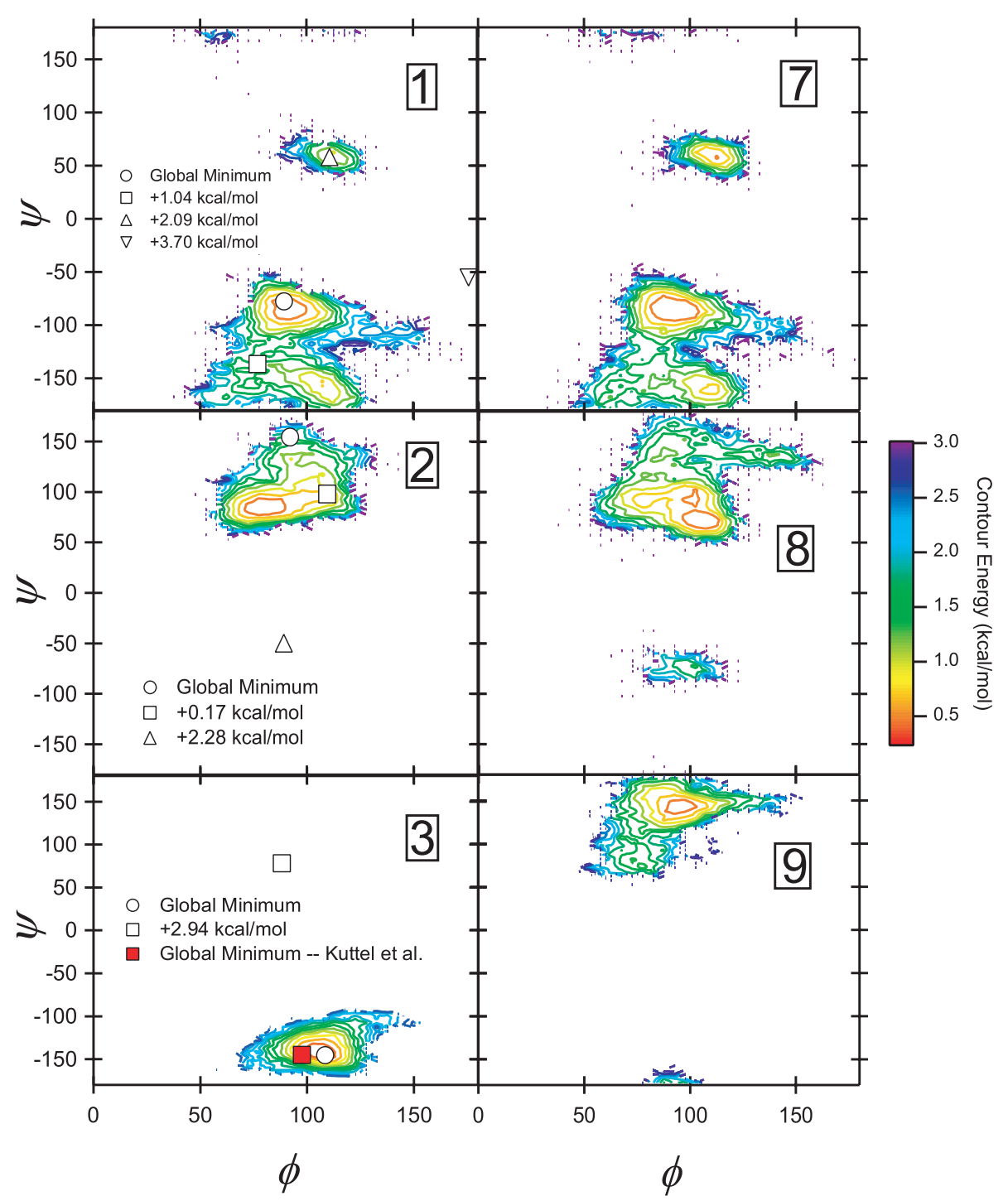

Figure 2: Relative free energy surfaces of all $\alpha$ linked models indicated by model number. Contour interval is $0.25 \mathrm{kcal} / \mathrm{mol}$ and the energy with respect to the global minima is also indicated in the color scale. Sections of the $\phi / \psi$ plot that lack contours indicate those portions of the PMF surface that are unpopulated at $300 \mathrm{~K}$ and are $\geq 3.0 \mathrm{kcal} / \mathrm{mol}$ higher in energy than the global minimum. Minima on PMF plots of glucose dimers (models 1,2 and 3 ) with open symbols are from the vacuum adiabatic map of Mendonca et al. ${ }^{26}$ Red square on model 3 is from PMF surface of Kuttel et al. ${ }^{42}$ 


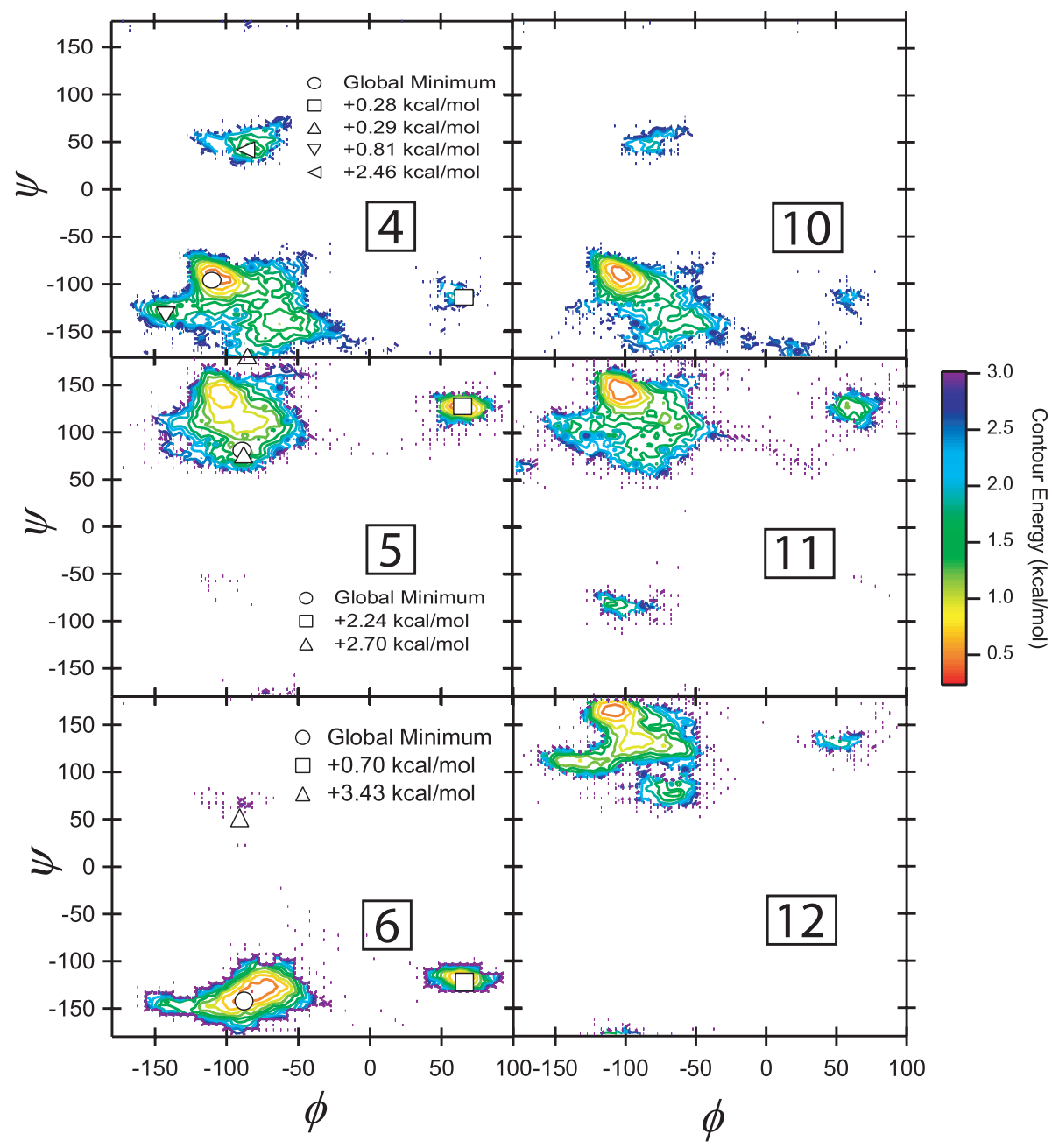

Figure 3: Relative free energy surfaces of all $\beta$ linked models indicated by model number. Contour interval is $0.25 \mathrm{kcal} / \mathrm{mol}$ and the energy with respect to the global minima is also indicated in the color scale. Sections of the $\phi / \psi$ plot that lack contours indicate those portions of the PMF surface that are unpopulated at $300 \mathrm{~K}$ and are $\geq 3.0 \mathrm{kcal} / \mathrm{mol}$ higher in energy than the global minimum. Minima on PMF plots of glucose dimers (models 4, 5 and 6 ) are from the vacuum adiabatic map of Mendonca et al. ${ }^{26}$ Mendonca et al. minima for the $\beta$ linked models are calculated based on disaccharides with $\beta$ reducing monosaccharides 

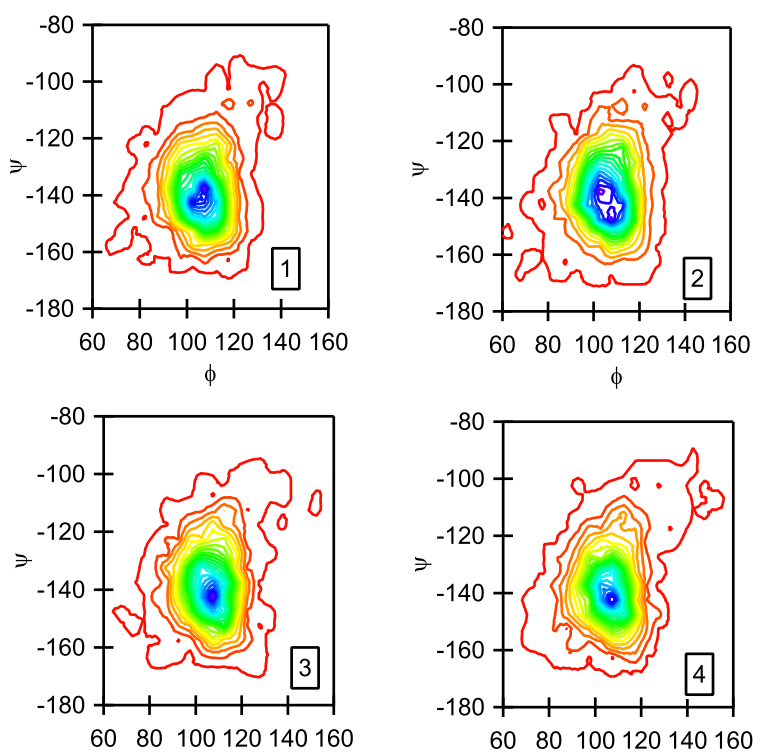

6080100120140160

$\phi$

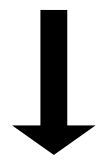

$\phi$

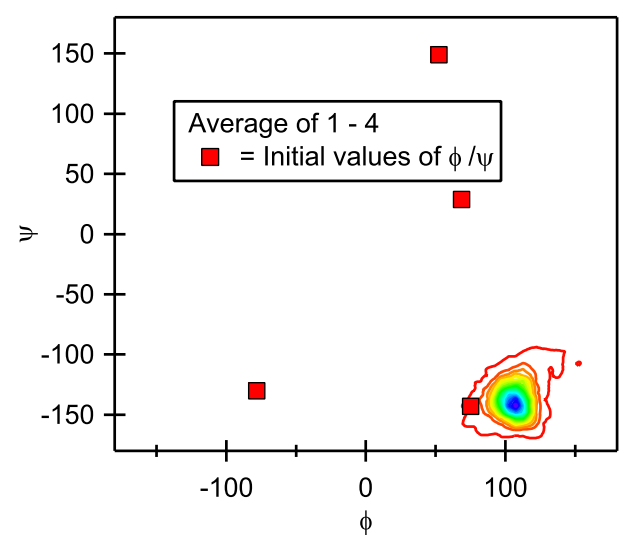

Figure 4: Normalized probability densities for four independent repMD simulations of model 3 , the resulting average and the four initial structures. Despite the widely spaced initial structure the four maps produced are equivalent. In each plot the contour interval and range of the contours are the same and run from red (relatively improbable) to purple (relatively probable). 


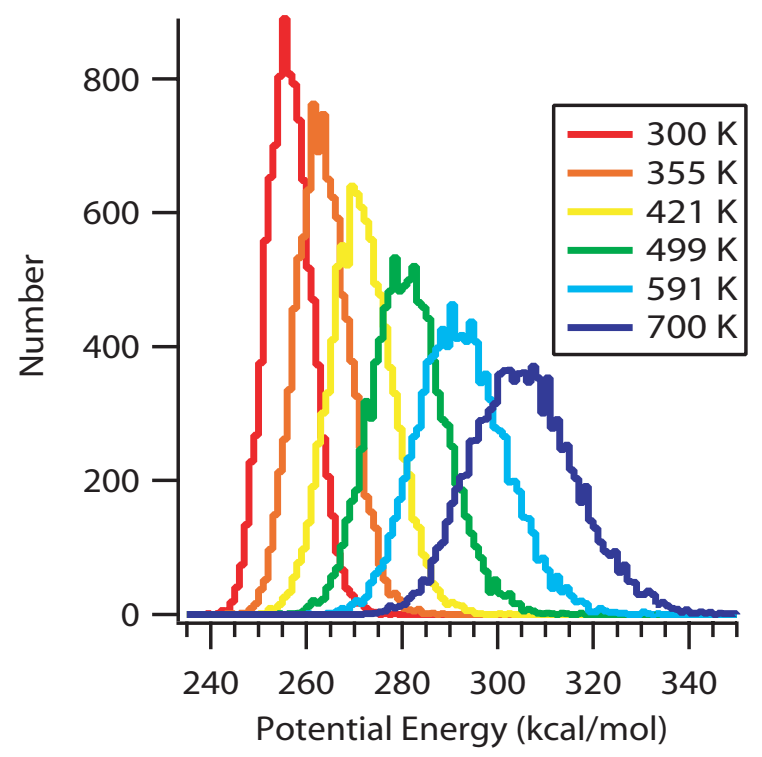

Figure 5: Representative potential energy histogram as a function of temperature for one repMD simulation (in this case of model 1). Each histogram has the expected gaussian shape and the substantial overlap between histograms suggests that acceptance ratios of replica swaps will be large enough to insure efficient searching of $\phi / \psi$ phase space. 

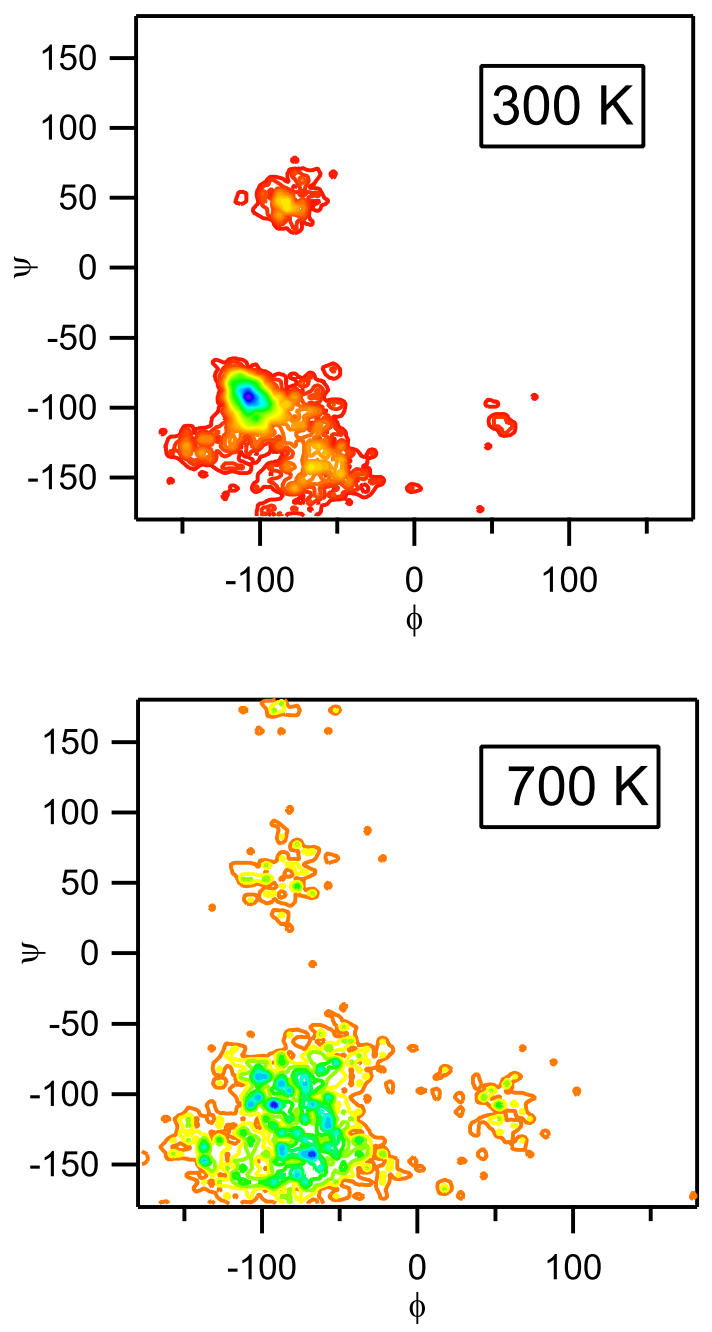

Figure 6: Normalized probability density for one repMD simulation of model 4 for the 300 and $700 \mathrm{~K}$ replicas. Probabilities are calculated using a 5 x 5 degree histogram. The comparison illustrates that at $700 \mathrm{~K}$ conformers with a wider range of $\phi / \psi$ exist with higher probability. For each plot the contour interval and range of contour values are the same. Contour values run from red (relatively improbable) to purple (relatively probable). 


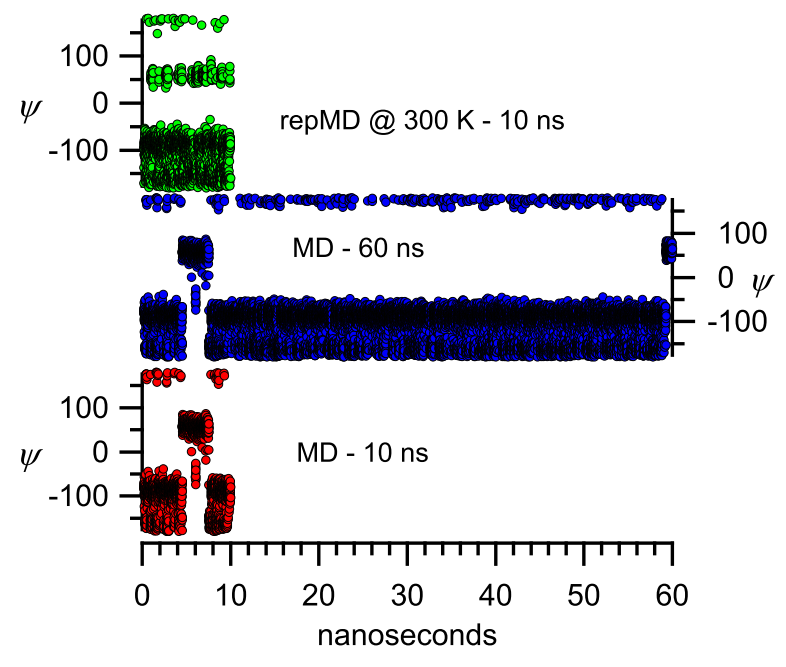

Figure 7: Comparison of sampling of $\psi$ for the $300 \mathrm{~K}$ replica of a repMD simulation of model 1 run for $10 \mathrm{~ns}$ and a conventional MD simulation, also of model 1, run for 10 and $60 \mathrm{~ns}$. All simulations are in the canonical ensemble and started from the same initial structure. Inspection suggests that sampling of the $\psi \approx 60^{\circ}$ conformer is far better in the $10 \mathrm{~ns}$ repMD simulation than in either the 10 or 60 ns conventional MD simulation. 


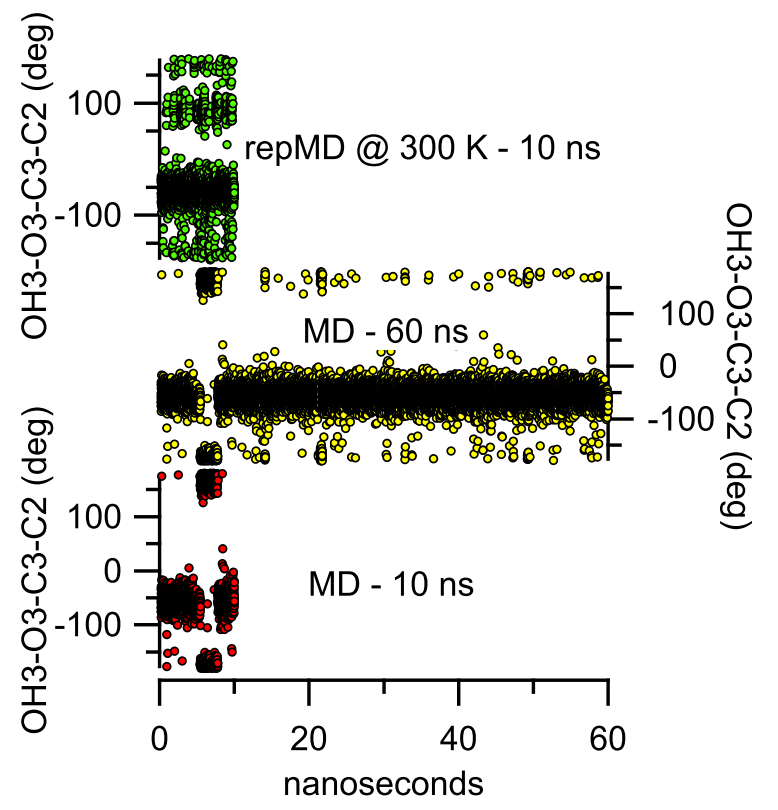

Figure 8: Comparison of sampling of the OH3-O3-C3-C2 dihedral angle for the $300 \mathrm{~K}$ replica of a repMD simulation of model 1 run for $10 \mathrm{~ns}$ and a conventional MD simulation, also of model 1, run for 10 and 60 ns. All simulations are in the canonical ensemble and started from the same initial structure. Sampling of this exocylic hydroxyl group is clearly enhanced in repMD, neither MD simulation ever samples the conformation at $100^{\circ}$. 


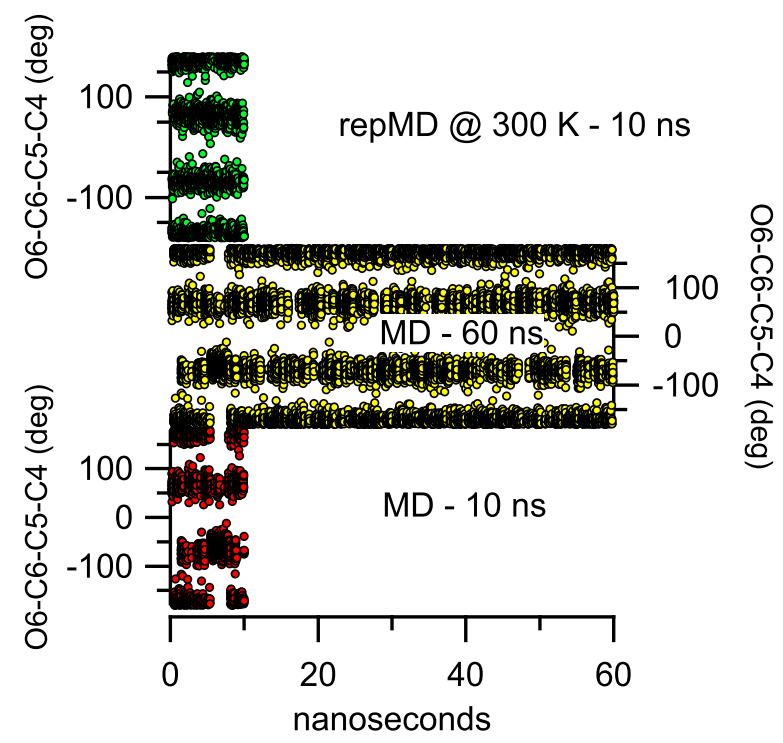

Figure 9: Comparison of sampling of hydroxymethyl for the $300 \mathrm{~K}$ replica of a repMD simulation of model 1 run for 10 ns and a conventional MD simulation, also of model 1, run for 10 and 60 ns. All simulations are in the canonical ensemble and started from the same initial structure. Sampling of the hydroxymethyl conformation is similar in conventional and repMD. 

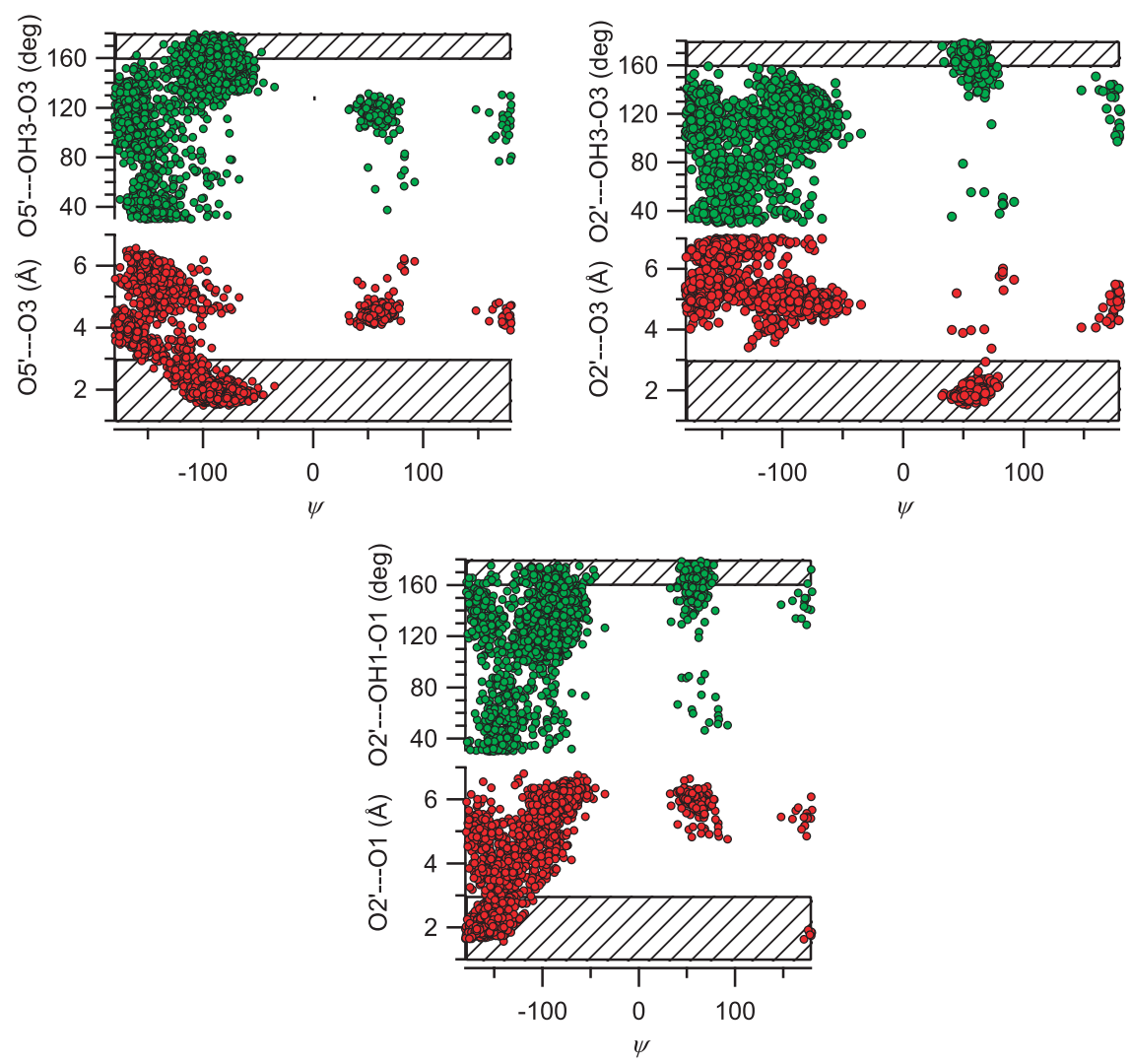

Figure 10: Hydrogen bond parameters for model 1 vs. $\psi$. 'Strong' hydrogen bond geometric parameters $\left(<3 \AA\right.$ in $\mathrm{O} \cdots \mathrm{O}$ distance and $>160^{\circ}$ in $\mathrm{O} \cdots \mathrm{H}-\mathrm{O}$ angle) are indicated by the cross hatched rectangles. These results suggest that the $\psi$ minimum centered at $\approx-100^{\circ}$ is stabilized by both a $\mathrm{O}^{\prime} \cdots \mathrm{OH} 3-\mathrm{O} 3$ and a ${ }^{\prime} 2^{\prime} \cdots \mathrm{OH} 1-\mathrm{O} 1$ hydrogen bond, while the minimum in $\psi$ centered at $\approx 60^{\circ}$ is stabilized only by a $\mathrm{O} 2^{\prime} \ldots \mathrm{OH} 3-\mathrm{O} 3$ interaction. 

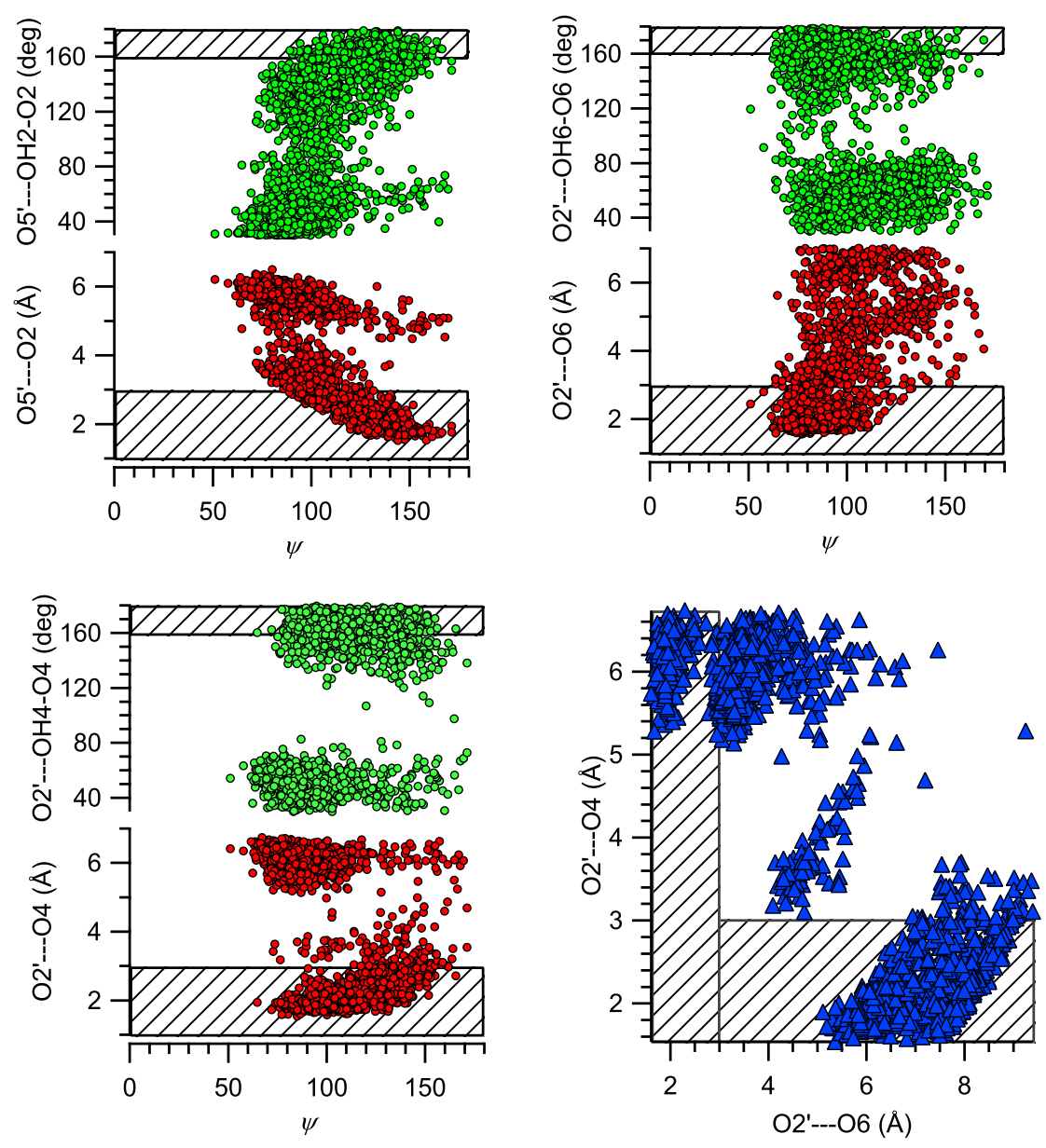

Figure 11: Hydrogen bond parameters for model 2 vs. $\psi$. 'Strong' hydrogen bond geometric parameters $\left(<3 \AA\right.$ in $\mathrm{O} \cdots \mathrm{O}$ distance and $>160^{\circ}$ in $\mathrm{O} \cdots \mathrm{H}-\mathrm{O}$ angle $)$ are indicated by the cross hatched rectangles. The minimum in $\psi$ centered at $\approx 100$ is predominantly stabilized by both a $\mathrm{O} 2{ }^{\prime} \cdots \mathrm{OH} 6-\mathrm{O} 6$ and a $\mathrm{O} 2^{\prime} \cdots \mathrm{OH} 4-\mathrm{O} 4$ hydrogen bond. The stabilization of conformers by multiple hydrogen bonding interactions involving a single donor or acceptor is not accounted for in the vacuum adiabatic map. The lower right hand panel (blue triangles) demonstrates that the hydrogen bonds $\mathrm{O} 2^{\prime} \cdots \mathrm{OH} 6-\mathrm{O} 6$ and a $\mathrm{O}^{\prime}{ }^{\prime} \cdots \mathrm{OH} 4-\mathrm{O} 4$ are distinct: they are not a single bifurcated hydrogen bond. 

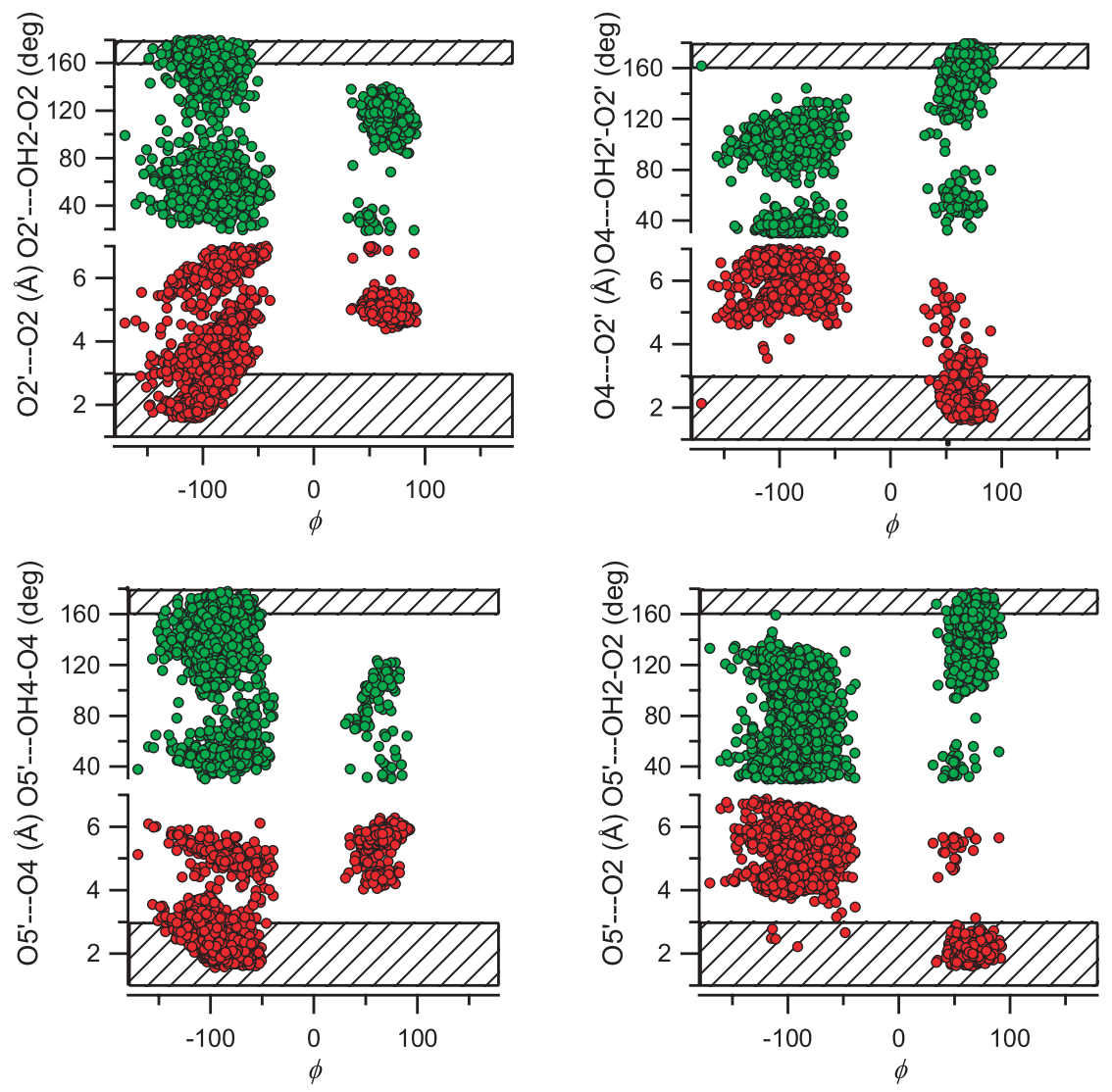

Figure 12: Hydrogen bond parameters for model 5 vs. $\phi$. 'Strong' hydrogen bond geometric parameters $\left(<3 \AA\right.$ in $\mathrm{O} \cdots \mathrm{O}$ distance and $>160^{\circ}$ in $\mathrm{O} \cdots \mathrm{H}-\mathrm{O}$ angle $)$ are indicated by the cross hatched rectangles. Hydrogen bonds stabilizing the minimum at $\phi \approx 75^{\circ}$ (in the right hand column) involve groups adjacent to the $(1 \rightarrow 3)$ linkage. 